\title{
Composite Fermions in Medium: Extending the Lipkin Model
}

\author{
S. Liebing* ${ }^{* 1}$ and D. Blaschke $e^{\dagger, \ddagger}$ \\ * Institute of Theoretical Physics, TU Bergakademie Freiberg, Freiberg, Germany \\ $\dagger$ Uniwersytet Wrocławski, Instytut Fizyki Teoretycznej, Wrocław, Poland \\ $\ddagger$ Joint Institute for Nuclear Research, 141980 Dubna, Russia
}

\begin{abstract}
The role of phase space occupation effects for the formation of two- and threeparticle bound states in a dense medium is investigated within an algebraic approach suitable for systems with short-range interactions. It is shown that for two-fermion bound states due to the account of the exchange symmetry (phase space occupation) effect (Pauli blocking) in a dense medium the binding energy is reduced and vanishes at a critical density (Mott effect). For three-fermion bound states, within a Faddeev equation approach, the intermediate formation of pair correlations leads to the representation as a suitably symmetrized fermion-boson bound state. It is shown that the Bose enhancement of fermion pairs can partially compensate the Pauli blocking between the fermions. This leads to the general result obtained by algebraic methods: three-fermion bound states in a medium with high phase space occupation appear necessarily as Borromean states beyond the Mott density of the two-fermion bound state.
\end{abstract}

\section{Introduction}

From the book of Lipkin [1] we know that in contrast to elementary bosons the deuterons (and other two-fermion bound states) being composite bosons localized in space require a Fourier spectrum of plane waves describing their nucleonic constituents which blocks a part of the fermionic phase space according to the Pauli principle. Contrary to naive expectation this leads to a statistical repulsion between bosons at high densities. This contribution discusses the question: what changes when a system of composite fermions is considered which consists of a boson-fermion pair where the boson is composed of two fermions? We provide an argument based on the algebraic treatment of these bound states that composite fermions are likely to form Borromean states [2] in a dense medium, since Pauli blocking shall make the

\footnotetext{
${ }^{1}$ Email: liebings@physik.tu-freiberg.de
} 
fermion pair an unbound correlation with a Bose enhancement factor which partly compensates for the Pauli blocking of the valence fermion so that the composite fermion remains a bound state.

We investigate bound states in many-particle systems and their stability under conditions of high phase space occupation. This situation is particularly prominent at low temperatures where we face a competition between Bose-Einstein condensation of bosonic two-particle bound states and BCS condensation of Cooper pairs. The point there is that the Mott effect due to Pauli blocking drives the binding energy to zero. Nevertheless, there is a strong resonant two-particle correlation in the scattering state spectrum above the continuum edge. This is general for two-particle correlations in Fermi systems, e.g., excitons in an electron-hole plasma [3], hydrogen in a non-ideal electron-proton plasma [4], deuterons in nuclear matter [5], mesons and diquarks in quark matter [6, 7].

This work considers also the question of three-particle bound states in a medium, examples being superfluid ${ }^{3} \mathrm{He}$, Tritons $/{ }^{3} \mathrm{He}$ in nuclear matter (sun, stars) and nucleons in quark matter. One important point there is also the role of Borromean states were the two-particle system is already unbound while the three-particle state remains bound at the same time. Here we will study the specific case that the two-particle state is bound when isolated but can undergo density ionization/dissociation (Mott effect) in a medium with high phase space occupation due to Pauli blocking. The question arises what this entails for the three-particle bound state. We demonstrate under very general conditions (basically only that the system is bound by short range interactions such as nuclear force) by using algebraic methods that the three-particle bound state has necessarily to persist as a bound state at densities exceeding the Mott density of the two-particle state. This effect of an "in-medium Borromean state" will be the stronger the stronger the pairing correlation in the three-particle state is.

\section{Composite Bosons}

In the beginning we consider elementary fermions coupled together to form composite bosons. The creation operator for such a boson resembles the subsequent application of two fermion creation operators. This can be represented as a linear combination of two plane wave functions (1). Thereby $r_{i}$ are the coordinates of the nucleons, $R$ is the center of mass and $r$ the relative coordinate of the composite particle (deuteron). In the same way we define the boson creation operator $D_{2 K}^{\dagger}$ and annihilation operator $D_{2 K^{\prime}}$ in 
momentum space.

$$
\begin{array}{r}
<R, r \mid D, 2 K>=e^{2 i K R} \phi(r)=\sum_{q} g_{q} e^{i(K+q) r_{1}} e^{i(K-q) r_{2}}, \\
D_{2 K}^{\dagger}=\sum_{q} g_{q} a_{(K+q) \uparrow}^{\dagger} a_{(K-q) \downarrow}^{\dagger}, D_{2 K^{\prime}}=\sum_{q} g_{q} a_{\left(K^{\prime}-q\right) \downarrow} a_{\left(K^{\prime}+q\right) \uparrow} .
\end{array}
$$

The next step in evaluating the properties of the created boson is to calculate the commutator between creation and annihilation operator. In contrast to elementary bosons the commutator does not vanish

$$
\begin{aligned}
{\left[D_{2 K^{\prime}}, D_{2 K}^{\dagger}\right]=} & \delta_{K K^{\prime}}-\Delta_{K K^{\prime}} \\
\Delta_{K K^{\prime}}= & \sum_{q} g_{q}\left\{g_{\left(K^{\prime}-K+q\right)} a_{\left(2 K-K^{\prime}-q\right) \downarrow}^{\dagger} a_{\left(K^{\prime}-q\right) \downarrow}\right. \\
& \left.+g_{\left(K^{\prime}-K-q\right)} a_{\left(2 K-K^{\prime}+q\right) \uparrow}^{\dagger} a_{\left(K^{\prime}+q\right) \uparrow}\right\} \\
\Delta_{K K}= & \sum_{q} g_{K-q}^{2} n_{q \downarrow}+g_{q-K}^{2} n_{q \uparrow} .
\end{aligned}
$$

The violation of the elementary Bose commutator is proportional to the occupation numbers of the fermionic constituents. For investigating phase space occupation effects in a system of $m$ composite bosons we apply a quasispin formalism. After doing so we apply a simple interaction operator $V=-\epsilon_{D} N_{D}$ to the multiparticle state just created. Thereby $N_{D}$ is the boson number operator, and the strength of the two-particle interaction is $G=\frac{-\epsilon_{D}}{\Omega}$, with $\Omega$ being the volume of the phase space and $-\epsilon_{D}$ being an eigenvalue of the interaction operator $V$

$$
\begin{aligned}
V & \left(D_{2 K}^{\dagger}\right)^{m}|0\rangle \\
& =-\left(\frac{\epsilon_{D}}{\Omega}\right)\left(\frac{\Omega}{2}\left(\frac{\Omega}{2}+1\right)-\left(\frac{2 m-\Omega}{2}\right)^{2}+\frac{2 m-\Omega}{2}\right)\left(D_{2 K}^{\dagger}\right)^{m}|0\rangle \\
& =-m \epsilon_{D}\left(1-\frac{m-1}{\Omega}\right)\left(D_{2 K}^{\dagger}\right)^{m}|0\rangle .
\end{aligned}
$$

Equation (6) can be evaluated for different values of $\Omega$ and $\epsilon_{D}$. The result is shown in figure 1. For low particle densities the binding energy increases due to Bose enhancement. By adding more particles the phase space gets filled up and it starts to cost energy to add more particles. This is due to the Pauli blocking of the fermionic substructure. 


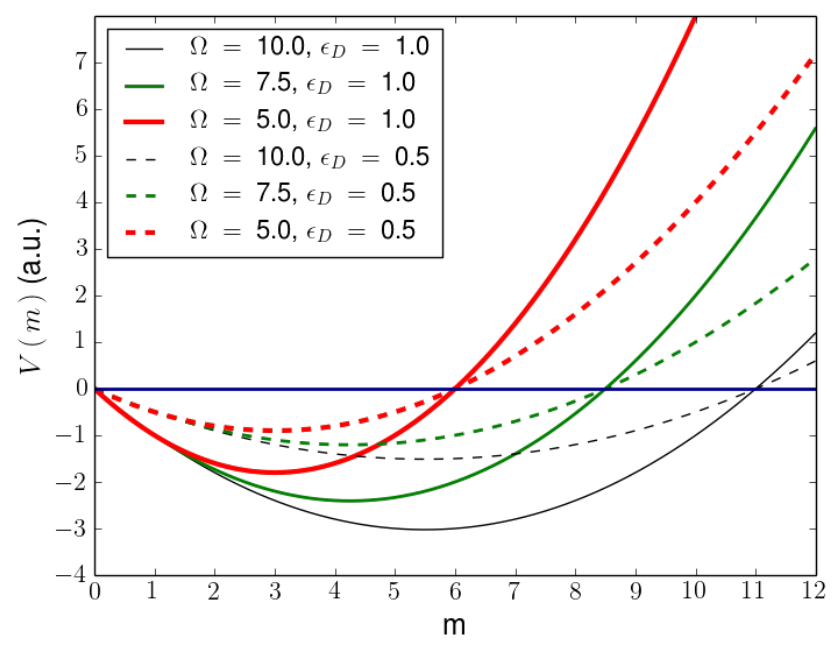

Figure 1: The figure shows how Bose enhancement evolves with growing particle density and gets overcompensated by the Pauli blocking. Here $\Omega$ is the spatial volume and $\epsilon_{D}$ the eigenvalue of the interaction operator $V$.

\section{Composite Fermions (Baryons)}

Similar to the algebraic treatment of composite bosons we can also consider baryons as composite fermions represented by creation and annihilation operators with one fermion with one boson operator which stand for the operators of quarks and diquarks, respectively

$$
N_{K}^{\dagger}=\sum_{q} g_{q} a_{\frac{K}{2}+q}^{\dagger} b_{\frac{K}{2}-q}^{\dagger}, N_{K^{\prime}}=\sum_{q} g_{q} b_{\frac{K^{\prime}}{2}-q^{\prime}} a_{\frac{K^{\prime}}{2}+q} .
$$

For these composite operators we calculate the anticommutator between composite fermions which, similar to the case of the composite bosons receives a correction term beyond the Kronecker symbol

$$
\begin{aligned}
{\left[N_{K^{\prime}}, N_{K}^{\dagger}\right] } & =\sum_{q} g_{q}\left(b_{\frac{K^{\prime}}{2}-q} a_{\frac{K^{\prime}}{2}+q} N_{K}^{\dagger}+N_{K}^{\dagger} b_{\frac{K^{\prime}}{2}-q^{\prime}} a_{\frac{K^{\prime}}{2}+q}\right) \\
& =\delta_{K, K^{\prime}}+\Delta_{K, K^{\prime}} \\
\Delta_{K, K^{\prime}} & =\sum_{q} g_{q}\left(g_{\frac{K-K^{\prime}}{2}-q} b_{K-\frac{K^{\prime}}{2}-q}^{\dagger} b_{\frac{K^{\prime}}{2}-q}-g_{\frac{K-K^{\prime}}{2}+q} a_{K-\frac{K^{\prime}}{2}+q}^{\dagger} a_{\frac{K^{\prime}}{2}+q}\right)
\end{aligned}
$$


For equal moments $K^{\prime} \rightarrow K$ this correction term takes the form

$$
\Delta_{K, K}=\sum_{q} g_{q}^{2}\left(n_{\frac{K}{2}-q}^{(D)}-n_{\frac{K}{2}+q}^{(Q)}\right)
$$

which makes the different phase space occupation effects for bosonic (D) and fermionic (Q) constituents apparent. In contrast to the composite boson case we observe competing phase space occupation effects: the Pauli blocking of quarks can be partially compensated by Bose enhancement of diquarks. This agrees with recent calculations of baryon properties in the PNJL model [8, 9]. In particular, baryons appear to be bound under conditions when diquarks are already unbound due to Pauli blocking (in-medium borromean state).

Two fermions are not allowed to be in the same quantum state therefore it is not correct to go to the limit $K^{\prime} \rightarrow K$. This was only done in order to investigate the effects qualitatively. For a systematic study of the properties of composite fermions or multi-fermion states it is required to deal with the case $K \neq K^{\prime}$. Then it is not immediately possible to express $\Delta_{K^{\prime} K}$ in terms of densities.

To quantify the effect it is required to develop a method to investigate the interactions between composite particles. To this end cluster virial expansion techniques will be developed for the case of nonideal plasmas with multiparticle correlations [10]. This goes beyond the scope of the present work and will be addressed in a forthcoming publication.

\section{Conclusions}

The algebraic treatment of a system of many composite bosons by Lipkin was reproduced and the results were visualized. Additionally, the approach was applied to describe composite fermions. We introduced creation and annihilation operators for a boson-fermion pair. The calculation of their anticommutator shows two competing density effects identified as Bose enhancement and Pauli blocking for the composite fermion. Consequently, there is a partial compensation which stabilizes the composite fermion in the medium. This can lead to the existence of Borromean states where the two-particle state is unbound while the three-particle state stays bound. Such kind of behavior was already discussed within the PNJL model [9]. Quantifying this effect in the presented approach requires the calculation of multi-fermion states, which goes beyond the focus of the current work. 


\section{Acknowledgment}

This work was supported in part by the Heisenberg-Landau Programme, by the Polish NCN under grant no. UMO-2011/02/A/ST2/00306 and by the COST Action MP1304 "NewCompStar".

\section{References}

[1] Lipkin H. "Quantum Mechanics." (Weizmann Institute, Rehovot, 1973)

[2] Zhukov M.V., Danilin B.V., Fedorov D.V., Bang J.M., Thompson I.J., Vaagen J.S. "Bound state properties of Borromean Halo nuclei: He-6 and Li-11." Physics Reports 231, 151 (1993).

[3] Stolz H., Zimmermann, R. "Correlated pairs and a mass action law in two-component Fermi systems excitons in an electron-hole plasma." physica status solidi (b) 94, 135-146 (1979).

[4] Kremp D., Schlanges M., Kraeft W.-D. "Quantum Statistics of Nonideal Plasmas." (Springer, Heidelberg, 2005).

[5] Schmidt M., Röpke, G, Schulz, H. "Generalized Beth-Uhlenbeck approach for hot nuclear matter." Annals Physics 202, 57-99 (1990).

[6] Blaschke D., Buballa M., Dubinin A., Röpke G., Zablocki D. "Generalized Beth-Uhlenbeck approach to mesons and diquarks in hot, dense quark matter." Annals Physics 348, 228-255 (2014).

[7] Zablocki D., Blaschke D., Röpke G. "BEC-BCS Crossover in Strongly Interacting Matter." in: "Metal-to-Nonmetal Transitions." Redmer R., Holst B. (Editors) (Springer Berlin Heidelberg, 2010) pp. 63-84.

[8] Blanqier E. "Standard particles in the SU(3) Nambu-Jona-Lasinio model and the Polyakov-NJL model." J. Phys. G 38, 105003 (2011).

[9] Blaschke D., Buballa M., Dubinin A., Zablocki D. "(P)NJL model approach to diquarks and baryons in quark matter." Proceedings "XXII Baldin Seminar on High-Energy Physics Problems", Dubna (2014).

[10] Röpke G., Bastian N.-U., Blaschke D., Klähn T., Typel S., Wolter H.H. "Cluster virial expansion for nuclear matter within a quasiparticle statistical approach." Nuclear Physics A 897, 70 (2013). 